\title{
Stereotactic Radiotherapy: An Emerging Treatment for Spinal Metastases
}

\author{
Max Dahele, Michael G. Fehlings, Arjun Sahgal
}

\begin{abstract}
Aim: The purpose of this concise update is to describe the emerging treatment of stereotactic body radiotherapy (SBRT) for spinal metastases. Rationale: Spinal metastases are common and can present complex clinical challenges that conventional treatment cannot always meet satisfactorily. Examples include a history of prior irradiation at the same site or radio-resistant tumor histology. Stereotactic body radiotherapy makes it possible to deliver high doses of radiation with the aim of improving tumor control and palliation. It is increasingly being offered to selected patients including those requiring re-irradiation and post-operative treatment. Conclusion: It is important that specialists managing patients with spinal metastases are aware of the potential advantages of SBRT and how this can complement and extend existing treatment approaches, including spinal decompression and stabilization.
\end{abstract}

RÉSUMÉ: La radiothérapie stéréotaxique, un traitement émergeant des métastases spinales. Objectif : Le but de cette brève mise à jour était de décrire le traitement émergeant par la radiothérapie stéréotaxique corporelle (RTSC) pour le traitement des métastases spinales. Justification : Les métastases spinales sont fréquentes et peuvent constituer un défi clinique complexe parce qu'elles ne répondent pas toujours de façon satisfaisante au traitement conventionnel, telles une histoire d'irradiation antérieure au niveau du même site ou une histologie tumorale radiorésistante. La RTSC permet de livrer de hautes doses de radiations dans le but d'améliorer le contrôle de la tumeur et également dans un but palliatif. Elle est de plus en plus souvent offerte à des patients bien choisis, particulièrement à ceux qui ont besoin d'une nouvelle irradiation ou d'un traitement postopératoire. Conclusion : Il est important que les spécialistes qui traitent les patients porteurs de métastases spinales soient au courant des avantages potentiels de la RTSC et de la façon dont elle peut servir de complément et élargir les approches de traitement existants, dont la décompression spinale et la stabilisation.

Can J Neurol Sci. 2011; 38: 247-250

Spinal metastases occur in up to $40 \%$ of cancer patients, often cause back pain, and if untreated can result in the medical emergency of malignant epidural spinal cord compression. Conventional external beam radiotherapy (RT) treatments using short-course low-dose radiation, such as 8 Gray (Gy) in a single fraction or 20 Gy in five daily fractions, are aimed primarily at pain control. Radiotherapy is the most common treatment for spine metastases. However, selected patients with oncological spinal instability or neural compression should be considered for spinal surgery which is optimally combined with post-operative $\mathrm{RT}^{1,2}$

Stereotactic body radiotherapy (SBRT) has recently emerged as an alternative, more aggressive, treatment option to conventional RT for selected patients with spinal metastases. Stereotactic body radiotherapy has resulted from rapid technologic advances in radiation oncology, in particular intensity modulated radiotherapy (IMRT) and image-guided radiotherapy (IGRT). The former makes it possible to shape radiation dose around a small target while sparing adjacent critical structures, including central structures within the target perimeter, such as the spinal cord (Figure 1). The latter allows the radiation to be delivered with millimetre precision ${ }^{3}$. This means that the dose to the vertebral tumor can now be escalated whilst maintaining a safe dose-limit to the spinal cord ${ }^{4}$. The objective of radiation therapy, therefore, has shifted from one primarily aimed at pain relief to long-term local tumor control ${ }^{5}$.

\section{What is stereotactic spine radiotherapy?}

Spine SBRT typically refers to the precise delivery of one to five fractions of high dose radiation to the tumor while sparing the surrounding normal tissues, and doses have ranged from 16$24 \mathrm{~Gy}$ in a single fraction to $20-40 \mathrm{~Gy}$ in two to five fractions ${ }^{5}$. Figure 1 illustrates the fundamental difference between conventional RT and intensity modulated SBRT treatment plans. The highly shaped dose distribution generated by intensity

From the Department of Radiation Oncology (MD), VU University Medical Centre, Amsterdam, The Netherlands; Division of Neurosurgery and Spinal Program (MGF), Toronto Western Hospital; Department of Radiation Oncology (AS), Princess Margaret Hospital and Sunnybrook Odette Cancer Centre, University of Toronto, Toronto, Ontario, Canada.

Received August 11, 2010. Final Revisions Submitted September 29, 2010. Correspondence to: Max Dahele, Department of Radiation Oncology, VU University Medical Centre, De Boelelaan 1117, 1081 HV Amsterdam, The Netherlands. 


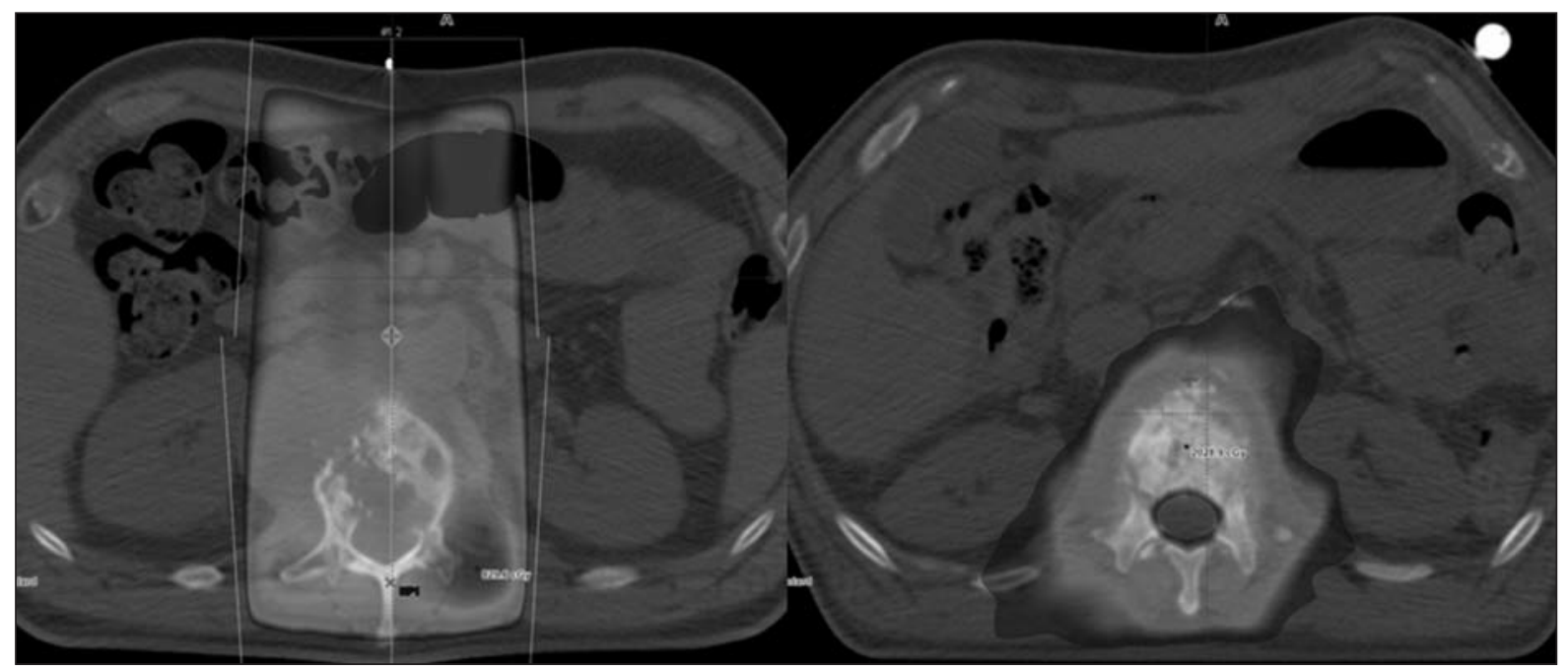

Figure 1: On the left is a conventional two-field radiotherapy treatment plan using anterior and posterior treatment beams. The entire target vertebra is treated to the prescribed dose of 8 Gy in a single fraction and both the spinal cord and anterior normal tissues in the path of the beams are exposed to the prescribed dose. On the right is an intensity-modulated stereotactic body radiotherapy (SBRT) treatment plan which also targeted the entire vertebra, however, in this instance it was treated with 16 Gy in a single fraction. The shaping of the dose is evident and, in particular, the relative sparing of the spinal canal which in this case receive less than $10 \mathrm{~Gy}$.

modulated radiation permits dose escalation within the target and a rapid dose fall-off beyond the target edges, enabling sparing of the surrounding normal tissues. Treatment volumes are typically limited to the gross tumor volume with or without part, or all, of the remaining vertebral segment. This is unlike conventional RT in which one or two simple RT fields are used to treat at least one healthy vertebral body above and below the disease, in order to ensure sufficient dose is delivered to the tumor. To meet the increased demands of accurate tumor delineation and identification of the organs-at-risk (e.g. the spinal cord, esophagus, lungs, kidneys), spine SBRT treatment planning places greater emphasis on imaging than conventional RT. Spine SBRT routinely requires both thin slice computed tomogram (CT) and magnetic resonance imaging (MRI) for treatment planning (Figure 2), and PET-CT and CT-myelography may also be helpful.

\section{How is stereotactic spine radiotherapy delivered?}

Computerized planning systems design and calculate the RT dose distribution using, for example, fixed-multiple field IMRT

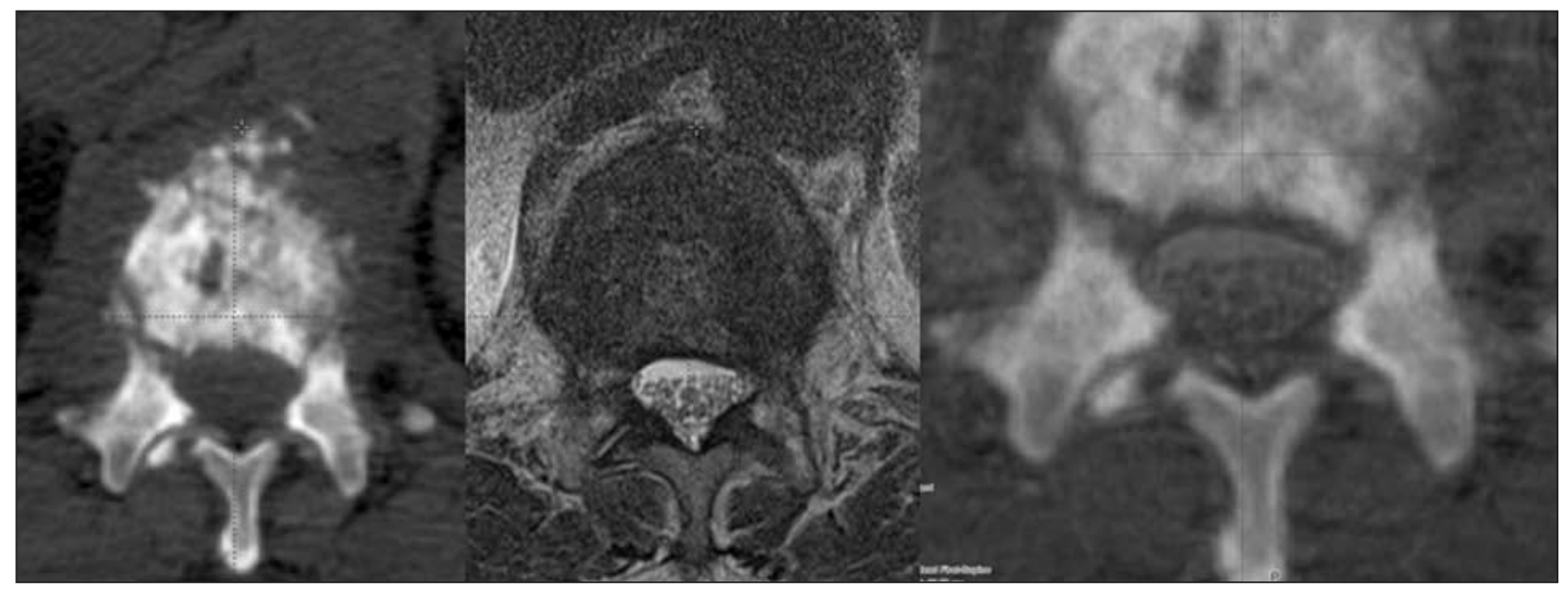

Figure 2: Radiotherapy planning requires that the tumor and normal tissues are accurately delineated. On the left is a CT image of the target and in the middle an axial T2-weighted MRI image at the same level. The cauda equina can be better visualized on the MRI. Fusing the images together (right) allows the benefits of both CT imaging for delineation of bony anatomy and MRI imaging for soft tissue anatomy such as the thecal sac and nerve roots to be used for treatment planning. 


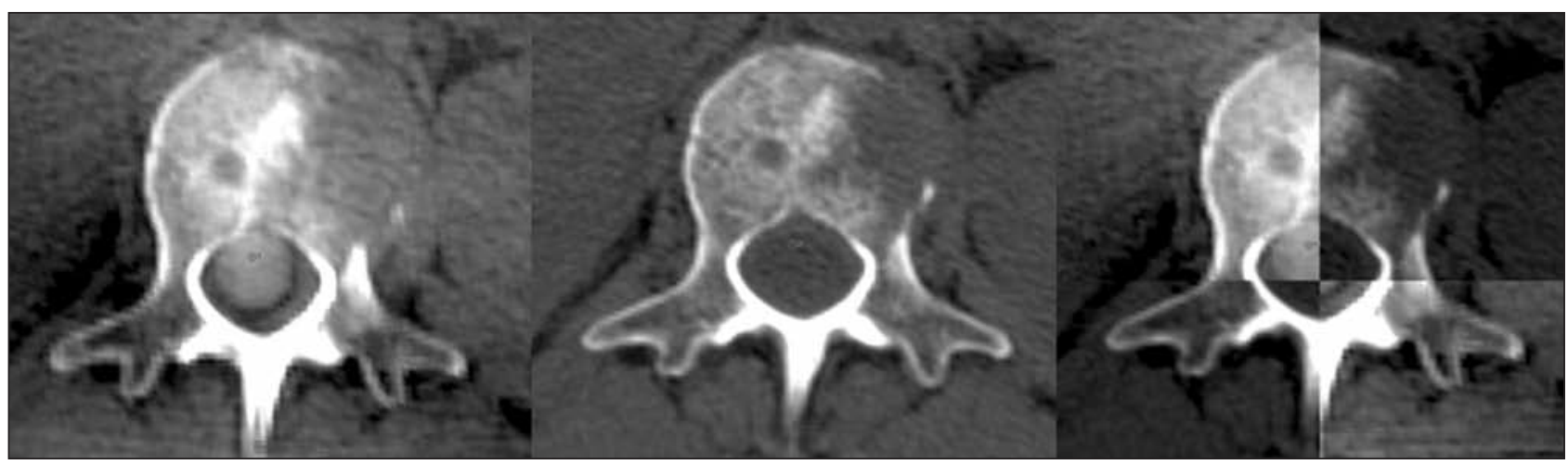

Figure 3: This figure illustrates the key features of cone-beam CT (CBCT) based imaged-guided radiotherapy (IGRT). On the left is a CBCT of the target taken just prior to radiation delivery to verify the target vertebrae position. The treatment planning CT scan originally taken at the time of radiation planning serves as the reference for positional matching, and is shown in the middle image. The CBCT is then fused to the reference planning CT images (right), and translated and rotated until they are well aligned. The noted positional deviations are then carried out by shifting the treatment couch. The correct position of the target may be verified, for example with another CBCT, and treatment delivery initiated.

or rotational IMRT (volumetric modulated arc therapy). To make it possible to deliver treatment with an overall accuracy of 1-2 $\mathrm{mm}$, IGRT is used to verify the position of the target and critical structures just prior to and during radiation delivery. One commonly used IGRT modality is cone-beam CT (CBCT). This consists of an isocentric fluoroscopic CT unit mounted on the linear accelerator (radiation delivery unit). An example of the IGRT process using CBCT is shown in Figure 3. Unlike x-rays, which image bony anatomy in two dimensions, CBCT provides three dimensional volumetric images of both bone and to some extent soft-tissues, thus aiding target and organ-at-risk localization for precise delivery.

\section{Patient selection for stereotactic spine radiotherapy and potential toxicities}

Spine SBRT is more complex and resource intensive than conventional RT with stringent requirements for treatment planning and delivery. Patient selection and multidisciplinary evaluation are important. Consultation with spine surgeons is often necessary to consider surgical options to correct critical neural compression or spinal instability before SBRT. Stereotactic body radiotherapy has most often been used for metastases less than three adjacent vertebral bodies in length, and when tumor is at least 1-2 mm away from the spinal cord in order to avoid under-dosing or missing epidural disease.

Although spine SBRT is usually well tolerated possible side effects include short-term fatigue, pain flare (a transient increase in pain shortly after radiotherapy), skin reactions and nausea. Important long-term consequences have been described following spine SBRT including vertebral compression fractures that may be associated with pain and, rarely, radiation myelopathy ${ }^{4}$ which at its worst can lead to irreversible paralysis.

\section{Current indications and evidence in support of spine SBRT}

Presently, spine SBRT is most often used in the treatment of patients with a good performance status/Karnofsky score, a limited extent of metastatic disease, and 'radio-resistant' tumor histology (e.g. renal cancer). This technique is also particularly useful in patients requiring re-irradiation, as there may be limited or no further options using conventional $\mathrm{RT}^{5}$.

The current evidence is limited to a growing body of retrospective single institution series and a few non-randomized prospective studies ${ }^{5}$. Initial rates of pain response and imagingbased local control are encouraging, and range anywhere from $80-95 \%$ in the primary, re-irradiation and post-operative scenarios $^{5,6}$. This compares with overall rates of pain response in bony metastases (not just spine) of approximately $60 \%$ for both single and multi-fraction conventional RT schedules ${ }^{7}$. Metastases with a defined paraspinal component (disease that has extended beyond the vertebrae) may stand to gain most from SBRT, as local control rates of only $46 \%$ at one-year and $39 \%$ at two-years have recently been reported with conventional RT alone . $^{8}$

Before making firmer conclusions we await with interest the outcome of the recently initiated randomized controlled study (RTOG 0631) comparing spine SBRT to conventional RT in patients with no prior radiation and symptomatic localized spinal metastases.

\section{Future developments}

Traditional surgery consists of an open invasive approach which is associated with significant post-operative morbidity. However, there have been recent developments in image-guided and minimally invasive spine surgery for metastases which include, for example, minimal access spine surgery using microsurgical techniques and percutaneous pedicle screw fixation or vertebral augmentation techniques such as vertebroplasty/kyphoplasty, that are aimed at reducing morbidity. Combining post-operative SBRT with new surgical approaches to achieve spinal cord decompression and mechanical stabilization not only allows for the goals of surgery to be met, but may also result in durable long-term tumor control.

Spine SBRT may also play an important role in patients with oligometastatic disease (e.g. five or fewer sites of metastasis throughout the body). It is thought that aggressive management 
of metastatic disease in these patients with ablative local therapies may result in long-term survival for selected patients. This is now an active area of investigation in cancer management ${ }^{9}$.

\section{Key points}

(1) There are limitations to using conventional RT for spinal metastases, in particular, for patients who require re-irradiation.

(2) Spine SBRT is an advanced treatment technique that allows spinal metastases to be treated to a higher dose than would otherwise be possible whilst respecting the tolerance of the surrounding critical normal tissues.

(3) The body of evidence supporting spine SBRT consists predominantly of heterogeneous patient populations and retrospective single institution series, however, overall spine SBRT has demonstrated promising efficacy and safety.

(4) Patients with neural structure compression or spinal instability should have a surgical opinion prior to deciding upon SBRT. ${ }^{10}$

(5) There is increasing interest in combining novel less invasive surgical approaches with spine SBRT.

\section{DisClosures}

Max Dahele: VU University Medical Centre has research collaborations with Varian Medical Systems, USA; BrainLAB AG, Germany and Velocity Medical Solutions, USA. Travel support from BrainLAB.

\section{REFERENCES}

1. Sciubba DM, Petteys RJ, Dekutoski MB, et al. Diagnosis and management of metastatic spine disease. J Neurosurg Spine. 2010;13:94-108.

2. Fisher CG, Dipaola CP, Ryken TC, et al. A novel classification system for spinal instability in neoplastic disease: an evidencebased approach and expert consensus from the spine oncology study group. Spine (Phila Pa 1976). 2010;35E1221-9.

3. Gerszten PC, Monaco EA, 3rd, Quader M, et al. Setup accuracy of spine radiosurgery using cone beam computed tomography image guidance in patients with spinal implants. J Neurosurg Spine. 2010;12:413-20.

4. Sahgal A, Ma L, Gibbs I, et al. Spinal cord tolerance for stereotactic body radiotherapy. Int J Radiat Oncol Biol Phys. 2010;77: 548-53.

5. Sahgal A, Larson DA, Chang EL. Stereotactic body radiosurgery for spinal metastases: a critical review. Int J Radiat Oncol Biol Phys. 2008;71:652-65.

6. Gerszten PC, Burton SA, Ozhasoglu C, Welch WC. Radiosurgery for spinal metastases: clinical experience in 500 cases from a single institution. Spine. 2007;32:193-9.

7. Sze WM, Shelley M, Held I, et al. Palliation of metastatic bone pain: single fraction versus multifraction radiotherapy - a systematic review of the randomised trials. Cochrane Database Syst Rev. 2004;(2):CD004721.

8. Mizumoto M, Harada $\mathrm{H}$, Asakura $\mathrm{H}$, et al. Radiotherapy for patients with metastases to the spinal column: a review of 603 patients at Shizuoka Cancer Center Hospital. Int J Radiat Oncol Biol Phys. 2011;79:208-13.

9. Milano MT, Katz AW, Muhs AG, et al. A prospective pilot study of curative-intent stereotactic body radiation therapy in patients with 5 or fewer oligometastatic lesions. Cancer. 2008;112:650-8.

10. Sahgal A, Bilsky M, Chang EL, et al. Stereotactic body radiotherapy for spinal metastases: current status, with a focus on its application in the postoperative patient. J Neurosurg Spine. 2010 Dec 24. [Epub ahead of print]. 